\title{
On the Gabor frame set for compactly supported continuous functions
}

\author{
Ole Christensen ${ }^{1}$, Hong Oh Kim² and Rae Young Kim³
}

\author{
Correspondence: rykim@ynu.ac.kr \\ ${ }^{3}$ Department of Mathematics, \\ Yeungnam University, 280 \\ Daehak-Ro, Gyeongsan, Gyeongbuk \\ 38541, Republic of Korea \\ Full list of author information is \\ available at the end of the article
}

\begin{abstract}
We identify a class of continuous compactly supported functions for which the known part of the Gabor frame set can be extended. At least for functions with support on an interval of length two, the curve determining the set touches the known obstructions. Easy verifiable sufficient conditions for a function to belong to the class are derived, and it is shown that the B-splines $B_{N}, N \geq 2$, and certain 'continuous and truncated' versions of several classical functions (e.g., the Gaussian and the two-sided exponential function) belong to the class. The sufficient conditions for the frame property guarantees the existence of a dual window with a prescribed size of the support.
\end{abstract}

MSC: $42 \mathrm{C} 15 ; 42 \mathrm{C} 40$

Keywords: Gabor frames; frame set; B-splines

\section{Introduction}

Frames is a functional analytic tool to obtain representations of the elements in a Hilbert space as a (typically infinite) superposition of building blocks. Frames indeed lead to decompositions that are similar to those obtained via orthonormal bases, but with much greater flexibility, due to the fact that the definition is significantly less restrictive. For example, in contrast to the case for a basis, the elements in a frame are not necessarily (linearly) independent, that is, frames can be redundant.

One of the main manifestations of frame theory is within Gabor analysis, where the aim is to obtain efficient representations of signals in a way that reflects the time-frequency distribution. For any $a, b>0$, consider the translation operator $T_{a}$ and the modulation operator $E_{b}$, both acting on the particular Hilbert space $L^{2}(\mathbb{R})$, given by $T_{a} f(x)=$ $f(x-a)$ and $E_{b} f(x)=e^{2 \pi i b x} f(x)$, respectively. Given $g \in L^{2}(\mathbb{R})$, the collection of functions $\left\{E_{m b} T_{n a} g\right\}_{m, n \in \mathbb{Z}}$ is called a (Gabor) frame if there exist constants $A, B>0$ such that

$$
A\|f\|^{2} \leq \sum_{m, n \in \mathbb{Z}}\left|\left\langle f, E_{m b} T_{n a} g\right\rangle\right|^{2} \leq B\|f\|^{2}, \quad \forall f \in L^{2}(\mathbb{R}) .
$$

If at least the upper condition is satisfied, $\left\{E_{m b} T_{n a} g\right\}_{m, n \in \mathbb{Z}}$ is called a Bessel sequence. It is known that for every frame $\left\{E_{m b} T_{n a} g\right\}_{m, n \in \mathbb{Z}}$, there exists a dual frame $\left\{E_{m b} T_{n a} h\right\}_{m, n \in \mathbb{Z}}$ such

(c) 2016 Christensen et al. This article is distributed under the terms of the Creative Commons Attribution 4.0 International License (http://creativecommons.org/licenses/by/4.0/), which permits unrestricted use, distribution, and reproduction in any medium, provided you give appropriate credit to the original author(s) and the source, provide a link to the Creative Commons license, and indicate if changes were made. 
that each $f \in L^{2}(\mathbb{R})$ has the decomposition

$$
f=\sum_{m, n \in \mathbb{Z}}\left\langle f, E_{m b} T_{n a} h\right\rangle E_{m b} T_{n a} h .
$$

The problem of determining $g \in L^{2}(\mathbb{R})$ and parameters $a, b>0$ such that $\left\{E_{m b} T_{n a} g\right\}_{m, n \in \mathbb{Z}}$ is a frame has attracted a lot of attention over the past 25 years. The frame set for a function $g \in L^{2}(\mathbb{R})$ is defined as the set

$$
\mathcal{F}_{g}:=\left\{(a, b) \in \mathbb{R}_{+}^{2} \mid\left\{E_{m b} T_{n a} g\right\}_{m, n \in \mathbb{Z}} \text { is a frame for } L^{2}(\mathbb{R})\right\}
$$

Clearly, the 'size' of the set $\mathcal{F}_{g}$ reflects the flexibility of the function $g$ in regard of obtaining expansions of type (1.1). In particular, it is known that $a b \leq 1$ is necessary for $\left\{E_{m b} T_{n a} g\right\}_{m, n \in \mathbb{Z}}$ to be a frame and that the number $(a b)^{-1}$ is a measure of the redundance of the frame; the smaller the number, the more redundant the frame. Thus, a reasonable function $g$ should lead to a frame $\left\{E_{m b} T_{n a} g\right\}_{m, n \in \mathbb{Z}}$ for values $(a b)^{-1}$ that are reasonably close to one. We remark that $\mathcal{F}_{g}$ is known to be open if $g$ belongs to the Feichtinger algebra; see $[1,2]$.

Until recently, the exact frame set was only known for very few functions: the Gaus$\operatorname{sian} g(x)=e^{-x^{2}}$ [3-5], the hyperbolic secant [6], and the functions $h(x)=e^{-|x|}, k(x)=$ $e^{-x} \chi_{[0, \infty[}(x)[7,8]$. In [9] a characterization was obtained for the class of totally positive functions of finite type, and based on [10], the frame set for functions $\chi_{[0, c]}, c>0$, was characterized in [11].

For applications of Gabor frames, it is essential that the window $g$ is a continuous function with compact support. Most of the related literature deals with special types of functions like truncated trigonometric functions or various types of splines; see [12-15]. Various classes of functions have also been considered, for example, functions yielding a partition of unity [16, 17], functions with short support or a finite number of sign-changes [18-20], or functions that are bounded away from zero on a specified part of the support [21]. The case of B-spline generated Gabor systems has attracted special attention; see, for example, [14, 20-23]. Interesting results in the rational case are obtained in [24].

To the best of our knowledge, the frame set has not been characterized for any function $g \in C_{c}(\mathbb{R}) \backslash\{0\}$. We will, among others, consider a class of functions for which we can extend the known set of parameters $(a, b)$ yielding a Gabor frame. The class of functions contains the B-splines $B_{N}, N \geq 2$, and certain 'continuous and compactly supported variants' of the mentioned functions $g, h$ and other classical functions. Furthermore, the results guarantee the existence of dual windows with a support size given in terms of the translation parameter.

In the rest of this introduction, we will describe the relevant class of windows and their frame properties. Proofs of the frame properties are in Section 2, and easy verifiable conditions for a function to belong to the class are derived in Section 3.

Let us first collect some of the known results concerning frame properties for continuous compactly supported functions; (i) is classical, and we refer to [25] for a proof.

Proposition 1.1 Let $N>0$, and assume that $g: \mathbb{R} \rightarrow \mathbb{C}$ is a continuous function with supp $g \subseteq\left[-\frac{N}{2}, \frac{N}{2}\right]$. Then the following holds:

(i) If $\left\{E_{m b} T_{n a} g\right\}_{m, n \in \mathbb{Z}}$ is a frame, then $a b<1$ and $a<N$. 
(ii) [21] Assume that $0<a<N, 0<b \leq \frac{2}{N+a}$, and $\inf _{x \in\left[-\frac{a}{2}, \frac{a}{2}\right]}|g(x)|>0$. Then

$\left\{E_{m b} T_{n a} g\right\}_{m, n \in \mathbb{Z}}$ is a frame, and there is a unique dual window $h \in L^{2}(\mathbb{R})$ such that $\operatorname{supp} h=\left[-\frac{a}{2}, \frac{a}{2}\right]$.

(iii) [20] Assume that $\frac{N}{2} \leq a<N$ and $0<b<\frac{1}{a}$. If $\left.g(x)>0, x \in\right]-\frac{N}{2}$, $\frac{N}{2}$, then $\left\{E_{m b} T_{n a} g\right\}_{m, n \in \mathbb{Z}}$ is a frame.

We will now introduce the window class that will be used in the current paper; it is a subset of the set of functions $g$ considered in Proposition 1.1(iii). The definition is inspired by certain explicit estimates for B-splines given by Trebels and Steidl [26]; this point will be clear in Proposition 3.1. First, fix $N>0$ and $0<a<N$. Consider the first-order difference $\Delta_{a} f$ and the second-order difference $\Delta_{a}^{2} f$ given by

$$
\Delta_{a} f(x)=f(x)-f(x-a), \quad \Delta_{a}^{2} f(x)=f(x)-2 f(x-a)+f(x-2 a) .
$$

We define the window class as the set of functions

$$
V_{N, a}:=\left\{f \in C(\mathbb{R}) \mid \operatorname{supp} f=\left[-\frac{N}{2}, \frac{N}{2}\right], f \text { is real-valued and satisfies (A1)-(A3) }\right\},
$$

where

(A1) $f$ is symmetric around the origin;

(A2) $f$ is strictly increasing on $\left[-\frac{N}{2}, 0\right]$;

(A3) If $a<\frac{N}{3}$, then $\Delta_{a}^{2} f(x) \geq 0, x \in\left[-\frac{N}{2},-\frac{N}{4}+\frac{3 a}{4}\right]$; if $a \geq \frac{N}{3}$, then $\Delta_{a}^{2} f(x) \geq 0, x \in\left[-\frac{N}{2}, 0\right] \cup\left\{-\frac{N}{4}+\frac{3 a}{4}\right\}$.

Note that by the symmetry condition (A1) a function $f \in V_{N, a}$ is completely determined by its behavior for $x \in\left[-\frac{N}{2}, 0\right]$. If $a \geq \frac{N}{3}$, then the point $-\frac{N}{4}+\frac{3 a}{4}$ considered in (A3) is not contained in $\left[-\frac{N}{2}, 0\right]$; however, if desired, the symmetry condition allows us to formulate the condition $\Delta_{a}^{2} f\left(-\frac{N}{4}+\frac{3 a}{4}\right) \geq 0$ alternatively as

$$
f\left(\frac{N}{4}-\frac{3 a}{4}\right)-2 f\left(-\frac{N}{4}-\frac{a}{4}\right) \geq 0
$$

because the argument $x-2 a$ of the last term in the second-order difference is less than $-\frac{N}{2}$.

The definition of $V_{N, a}$ is technical, but we will derive easy verifiable conditions for a function $g$ to belong to this set in Proposition 3.1 and also provide several natural examples of such functions. Our main result extends the range of $b>0$ yielding a frame, compared with Proposition 1.1(ii):

Theorem 1.2 For $N>0$, let $0<a<N$ and $\frac{2}{N+a}<b \leq \frac{4}{N+3 a}$. Assume that $g \in V_{N, a}$. Then the Gabor system $\left\{E_{m b} T_{n a} g\right\}_{m, n \in \mathbb{Z}}$ is a frame for $L^{2}(\mathbb{R})$, and there is a unique dual window $h \in L^{2}(\mathbb{R})$ such that $\operatorname{supp} h \subseteq\left[-\frac{3 a}{2}, \frac{3 a}{2}\right]$.

For an illustration of Proposition 1.1 and Theorem 1.2, see Figure 1.

Membership of a function $g$ in a set $V_{N, a}$ for some $\left.a \in\right] 0, N$ [ only gives information about the frame properties of $\left\{E_{m b} T_{n a} g\right\}_{m, n \in \mathbb{Z}}$ for this specific value of the translation parameter $a$. In order to get an impression of the frame properties of $\left\{E_{m b} T_{n a} g\right\}_{m, n \in \mathbb{Z}}$ in a region in the $(a, b)$-plane, we need to consider a function $g$ that belongs to $V_{N, a}$ for an in- 


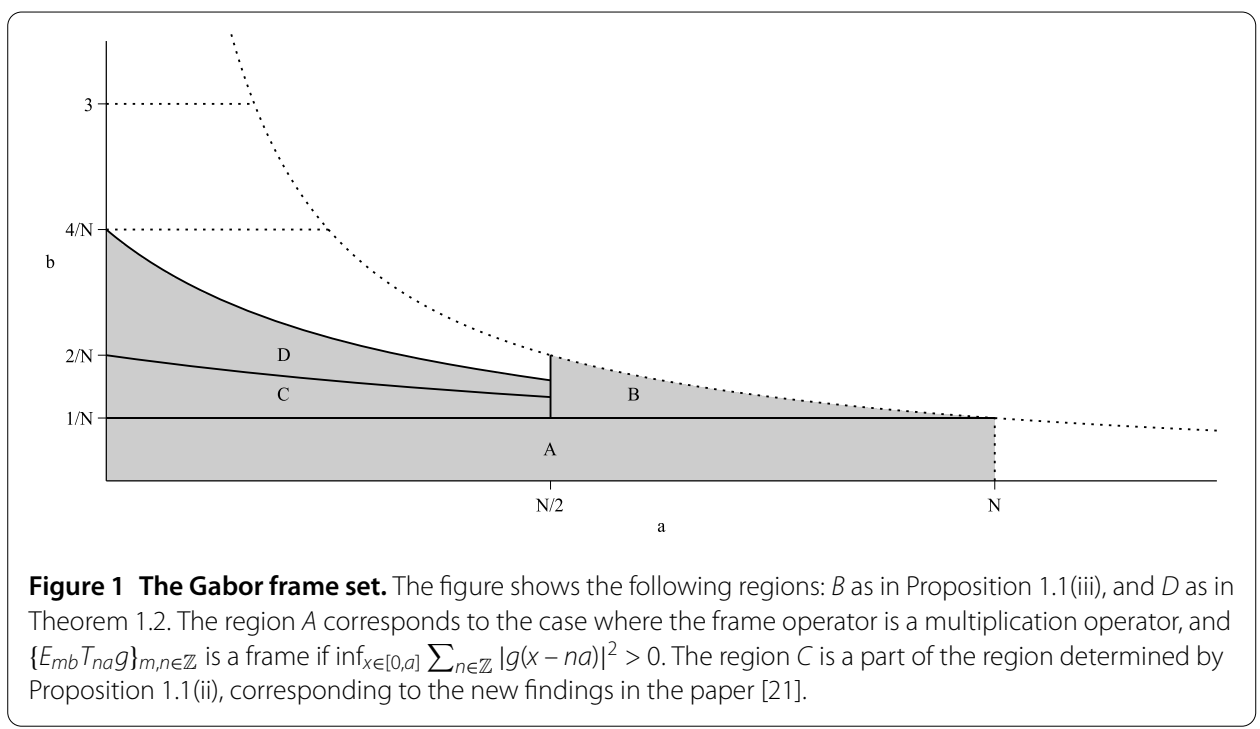

terval of $a$-values, preferably for all $a \in] 0, N[$. Fortunately, several natural functions have this property. The following list collects some of the results we will obtain in Section 3. Considering any $N \in \mathbb{N} \backslash\{1\}$,

- The B-spline $B_{N}$ of order $N$ belongs to $\bigcap_{0<a<N} V_{N, a}$;

- The function $f_{N}(x):=\cos ^{2 N-2}\left(\frac{\pi x}{N}\right) \chi_{\left[-\frac{N}{2}, \frac{N}{2}\right]}(x)$ belongs to $\bigcap_{0<a<N} V_{N, a}$;

- The function $h_{N}(x):=\left(e^{-|x|}-e^{-\frac{N}{2}}\right) \chi_{\left[-\frac{N}{2}, \frac{N}{2}\right]}(x)$ belongs to $\bigcap_{0<a<N} V_{N, a}$;

- The function $g_{N}(x):=\left(e^{-x^{2}}-e^{-\frac{N^{2}}{4}}\right) \chi_{\left[-\frac{N}{2}, \frac{N}{2}\right]}(x)$ belongs to $\bigcap_{\frac{3 N}{7} \leq a<N} V_{N, a}$.

In particular, Proposition 1.1 and Theorem 1.2 imply that for $N \in \mathbb{N} \backslash\{1\}$, the functions $B_{N}, f_{N}$, and $h_{N}$ generate frames whenever $0<a<N$ and $0<b \leq \frac{4}{N+3 a}$, and $g_{N}$ generates a frame whenever $\frac{3 N}{7} \leq a<N$ and $0<b \leq \frac{4}{N+3 a}$.

Note that the limit curve $b=\frac{4}{N+3 a}$ in Theorem 1.2 touches the known obstructions for Gabor frames. In fact, for $N=2$, we obtain that $b \rightarrow 2$ as $a \rightarrow 0$. Since it is known that the $\mathrm{B}$-spline $B_{2}$ does not generate a frame for $b=2[12,16]$, we cannot go beyond this. We also know that at least for some functions $g \in \bigcap_{0<a<N} V_{N, a}$, parts of the region determined by the inequalities $b<2, a<2, a b<1$ do not belong to the frame set. Considering, for example, the B-spline $B_{2}$ [21] shows that the point $(a, b)=\left(\frac{2}{7}, \frac{7}{4}\right)$ does not belong to the frame set. For $a=\frac{2}{7}$, Theorem 1.2 guarantees the frame property for $b<\frac{7}{5}$, which is close to the obstruction. These considerations indicate that the frame region in Theorem 1.2 in a quite accurate way describes the maximally possible frame set below $b=2$ that is valid for all the functions in $V_{N, a}$, at least for $N=2$.

\section{Frame properties for functions $g \in V_{N, a}$}

The purpose of this section is to prove Theorem 1.2. Since the functions $g \in V_{N, a}$ are bounded and have compact support, they generate Bessel sequences $\left\{E_{m b} T_{n a} g\right\}_{m, n \in \mathbb{Z}}$ for all $a, b>0$. By the duality conditions $[27,28]$ two bounded functions $g, h$ with compact support generate dual frames $\left\{E_{m b} T_{n a} g\right\}_{m, n \in \mathbb{Z}}$ and $\left\{E_{m b} T_{n a} h\right\}_{m, n \in \mathbb{Z}}$ for some fixed $a, b>0$ if and only if

$$
\sum_{m \in \mathbb{Z}} g(x-\ell / b+m a) \overline{h(x+m a)}=b \delta_{\ell, 0}, \quad \text { a.e. } x \in\left[-\frac{a}{2}, \frac{a}{2}\right]
$$


in particular, a function $g \in V_{N, a}$ and a bounded real-valued function $h$ with support on $\left[-\frac{3 a}{2}, \frac{3 a}{2}\right]$ generate dual Gabor frames $\left\{E_{m b} T_{n a} g\right\}_{m, n \in \mathbb{Z}}$ and $\left\{E_{m b} T_{n a} h\right\}_{m, n \in \mathbb{Z}}$ for $L^{2}(\mathbb{R})$ for some $b \leq \frac{4}{N+3 a}$ if and only if, for $\ell=0, \pm 1$,

$$
\sum_{m=-1}^{1} g(x-\ell / b+m a) h(x+m a)=b \delta_{\ell, 0}, \quad \text { a.e. } x \in\left[-\frac{a}{2}, \frac{a}{2}\right] .
$$

Given $g \in V_{N, a}$, we therefore consider the $3 \times 3$ matrix-valued function $G$ on $\left[-\frac{a}{2}, \frac{a}{2}\right]$ defined by

$$
G(x):=\left(g\left(x-\frac{\ell}{b}+m a\right)\right)_{-1 \leq \ell, m \leq 1}=\left(\begin{array}{ccc}
g\left(x+\frac{1}{b}-a\right) & g\left(x+\frac{1}{b}\right) & g\left(x+\frac{1}{b}+a\right) \\
g(x-a) & g(x) & g(x+a) \\
g\left(x-\frac{1}{b}-a\right) & g\left(x-\frac{1}{b}\right) & g\left(x-\frac{1}{b}+a\right)
\end{array}\right) .
$$

In terms of the $G(x)$, condition (2.1) simply means that

$$
G(x)\left(\begin{array}{c}
h(x-a) \\
h(x) \\
h(x+a)
\end{array}\right)=\left(\begin{array}{l}
0 \\
b \\
0
\end{array}\right), \quad \text { a.e. } x \in\left[-\frac{a}{2}, \frac{a}{2}\right] .
$$

We will show that the matrix $G(x)$ is invertible for all $x \in\left[-\frac{a}{2}, \frac{a}{2}\right]$; this will ultimately give us a bounded and compactly supported function $h$ satisfying (2.1) and hereby prove Theorem 1.2. The invertibility of $G(x)$ will be derived as a consequence of a series of lemmas, where we first consider $x \in\left[-\frac{a}{2}, 0\right]$. Note that the proof of the first result does not use property (A3).

Lemma 2.1 For $N>0$, let $0<a<N$ and $\frac{2}{N+a}<b \leq \frac{4}{N+3 a}$. Assume that $g \in V_{N, a}$ and let $x \in\left[-\frac{a}{2}, 0\right]$. Then the following hold:

(a) $g\left(x+\frac{1}{b}+a\right) \leq g\left(x+\frac{1}{b}\right)<g\left(x+\frac{1}{b}-a\right) \neq 0$;

(b) $g\left(x-\frac{1}{b}-a\right) \leq g\left(x-\frac{1}{b}\right)<g\left(x-\frac{1}{b}+a\right) \neq 0$;

(c) $g(x)>g(x-a)$ and $g(x) \geq g(x+a)$ with equality only for $x=-\frac{a}{2}$.

Proof For (a), let $x \in\left[-\frac{a}{2}, 0\right]$. Using $b \leq \frac{4}{N+3 a}$ and $a<N$, we have

$$
x+\frac{1}{b}-a \geq \frac{1}{b}-\frac{3 a}{2} \geq \frac{N+3 a}{4}-\frac{3 a}{2}>-\frac{N}{2} .
$$

It follows that

$$
\left.x+\frac{1}{b}-a \subseteq\left[\frac{1}{b}-\frac{3 a}{2}, \frac{1}{b}-a\right] \subseteq\right]-\frac{N}{2}, \frac{N}{2}[.
$$

Using that $b \leq \frac{4}{N+3 a}<\frac{1}{a}$, it follows that $-2 x-\frac{2}{b}+a \leq 2\left(a-\frac{1}{b}\right)<0$; thus,

$$
-x-\frac{1}{b}+a<x+\frac{1}{b}
$$

Since $g(x)>0$ for $x \in]-\frac{N}{2}, \frac{N}{2}$ [, we have by (2.3) that $g\left(x+\frac{1}{b}-a\right) \neq 0$. By (A2) we know that $g$ is strictly decreasing on [0, $\frac{N}{2}$ ]. If $x+\frac{1}{b}-a \leq 0$, then we have by (2.3), (2.4), and the 
symmetry of $g$ that

$$
g\left(x+\frac{1}{b}-a\right)=g\left(-x-\frac{1}{b}+a\right)>g\left(x+\frac{1}{b}\right) \geq g\left(x+\frac{1}{b}+a\right)
$$

if $x+\frac{1}{b}-a>0$, then we have $g\left(x+\frac{1}{b}-a\right)>g\left(x+\frac{1}{b}\right) \geq g\left(x+\frac{1}{b}+a\right)$. Hence, (a) holds. Similarly, (b) and (c) hold.

We now show that if $g \in V_{N, a}$ and $a \geq N / 3$, then condition (A3) automatically holds on a larger interval.

Lemma 2.2 For $N>0$, let $0<a<N$. Assume that $g \in V_{N, a}$. Then $\Delta_{a}^{2} g(x) \geq 0$ for all $x \in$ $\left[-\frac{N}{2},-\frac{N}{4}+\frac{3 a}{4}\right]$.

Proof It suffices to show that, for $a \geq \frac{N}{3}$,

$$
\Delta_{a}^{2} g(x) \geq 0, \quad \forall x \in\left[0,-\frac{N}{4}+\frac{3 a}{4}\right]
$$

We first note that (A1) and (A2) imply that, for $x \in\left[0,-\frac{N}{4}+\frac{3 a}{4}\right]$,

$$
g(x) \geq g\left(-\frac{N}{4}+\frac{3 a}{4}\right) \quad \text { and } \quad g(x-a) \leq g\left(-\frac{N}{4}-\frac{a}{4}\right) .
$$

For $a \geq \frac{N}{3}$, we have $-\frac{N}{4}-\frac{5 a}{4}<-\frac{N}{2}$; due to the compact support of $g, f\left(-\frac{N}{4}-\frac{5 a}{4}\right)=0$; thus using (A1) again, we have

$$
\begin{aligned}
\Delta_{a}^{2} g\left(-\frac{N}{4}+\frac{3 a}{4}\right) & =g\left(-\frac{N}{4}+\frac{3 a}{4}\right)-2 g\left(-\frac{N}{4}-\frac{a}{4}\right) \\
& =g\left(\frac{N}{4}-\frac{3 a}{4}\right)-2 g\left(-\frac{N}{4}-\frac{a}{4}\right) .
\end{aligned}
$$

Together with (2.5), this shows that

$$
0 \leq \Delta_{a}^{2} g\left(-\frac{N}{4}+\frac{3 a}{4}\right) \leq \Delta_{a}^{2} g(x), \quad \forall x \in\left[0,-\frac{N}{4}+\frac{3 a}{4}\right]
$$

as desired.

Let $G_{i j}(x)$ denote the $i j$ th minor of $G(x)$, the determinant of the submatrix obtained by removing the $i$ th row and the $j$ th column from $G(x)$.

Lemma 2.3 For $N>0$, let $0<a<N$ and $\frac{2}{N+a}<b \leq \frac{4}{N+3 a}$. Assume that $g \in V_{N, a}$ and let $x \in\left[-\frac{a}{2}, 0\right]$. Then the following hold:

(a) $G_{21}(x) \geq 0$, and equality holds iff $g\left(x+\frac{1}{b}\right)=g\left(x+\frac{1}{b}+a\right)=0$;

(b) $G_{23}(x) \geq 0$, and equality holds iff $g\left(x-\frac{1}{b}-a\right)=g\left(x-\frac{1}{b}\right)=0$;

(c) $G_{22}(x) \geq G_{21}(x)+G_{23}(x)$. 
Proof Since $g \geq 0$, (a) and (b) follow from Lemma 2.1(a), (b). For (c), we note that $\Delta_{a} g(x)=$ $g(x)-g(x-a)=g(-x)-g(-x+a)=-\Delta_{a} g(-x+a)$ by the symmetry of $g$. Now a direct calculation shows that

$$
\begin{aligned}
& G_{22}(x)-G_{21}(x)-G_{23}(x) \\
& =-\Delta_{a} g\left(x+\frac{1}{b}\right) \Delta_{a} g\left(x-\frac{1}{b}+a\right)+\Delta_{a} g\left(x+\frac{1}{b}+a\right) \Delta_{a} g\left(x-\frac{1}{b}\right) \\
& \quad=\Delta_{a} g\left(-x-\frac{1}{b}+a\right) \Delta_{a} g\left(x-\frac{1}{b}+a\right)-\Delta_{a} g\left(-x-\frac{1}{b}\right) \Delta_{a} g\left(x-\frac{1}{b}\right) .
\end{aligned}
$$

Hence, it suffices to show that

$$
\Delta_{a} g\left(x-\frac{1}{b}+a\right) \geq \Delta_{a} g\left(x-\frac{1}{b}\right), \quad x \in\left[-\frac{a}{2}, 0\right]
$$

and

$$
\Delta_{a} g\left(-x-\frac{1}{b}+a\right) \geq \Delta_{a} g\left(-x-\frac{1}{b}\right), \quad x \in\left[-\frac{a}{2}, 0\right]
$$

or, equivalently, that $\Delta_{a}^{2} g\left(x-\frac{1}{b}+a\right) \geq 0$ and $\Delta_{a}^{2} g\left(-x-\frac{1}{b}+a\right) \geq 0$, both for $x \in\left[-\frac{a}{2}, 0\right]$. Since $\left[-\frac{1}{b}+\frac{a}{2},-\frac{1}{b}+a\right] \cup\left[-\frac{1}{b}+a,-\frac{1}{b}+\frac{3 a}{2}\right]=\left[-\frac{1}{b}+\frac{a}{2},-\frac{1}{b}+\frac{3 a}{2}\right]$, this means precisely that

$$
\Delta_{a}^{2} g(x) \geq 0, \quad x \in\left[-\frac{1}{b}+\frac{a}{2},-\frac{1}{b}+\frac{3 a}{2}\right] .
$$

We note that, for $\frac{2}{N+a}<b \leq \frac{4}{N+3 a}$,

$$
-\frac{N}{2} \leq-\frac{1}{b}+\frac{a}{2},-\frac{1}{b}+\frac{3 a}{2} \leq-\frac{N}{4}+\frac{3 a}{4}
$$

Together with Lemma 2.2, this implies that (2.6) holds, as desired.

After this preparation, we can now show that $G(x)$ is indeed invertible for $x \in\left[-\frac{a}{2}, \frac{a}{2}\right]$ under the assumptions in Theorem 1.2.

Corollary 2.4 For $N>0$, let $0<a<N$ and $\frac{2}{N+a}<b \leq \frac{4}{N+3 a}$. Assume that $g \in V_{N, a}$. Then $\operatorname{det} G(x) \neq 0$ for $x \in\left[-\frac{a}{2}, \frac{a}{2}\right]$.

Proof First, consider $x \in\left[-\frac{a}{2}, 0\right]$. By Lemma 2.3(c) we have

$$
\begin{aligned}
\operatorname{det} G(x) & =-g(x-a) G_{21}(x)+g(x) G_{22}(x)-g(x+a) G_{23}(x) \\
& \geq-g(x-a) G_{21}(x)+g(x)\left(G_{21}(x)+G_{23}(x)\right)-g(x+a) G_{23}(x) \\
& =(g(x)-g(x-a)) G_{21}(x)+(g(x)-g(x+a)) G_{23}(x) \\
& =: A_{N}(x) .
\end{aligned}
$$

Using Lemma 2.1(c) and Lemma 2.3(a), (b), we have

$$
(g(x)-g(x-a)) G_{21}(x) \geq 0 \quad \text { and } \quad(g(x)-g(x+a)) G_{23}(x) \geq 0 .
$$


Thus, $A_{N}(x) \geq 0, x \in\left[-\frac{a}{2}, 0\right]$. If $A_{N}(x)>0$ for all $x \in\left[-\frac{a}{2}, 0\right]$, then the proof is completed; thus, the rest of the proof is focused on the case where $A_{N}\left(x_{0}\right)=0$ for some $x_{0} \in\left[-\frac{a}{2}, 0\right]$. In this case, Lemma 2.1(c) shows that either

$$
G_{21}\left(x_{0}\right)=0, \quad G_{23}\left(x_{0}\right)=0
$$

or

$$
G_{21}\left(x_{0}\right)=0, \quad x_{0}=-\frac{a}{2} .
$$

The case (2.8) actually cannot occur. Indeed, if $G_{21}\left(-\frac{a}{2}\right)=0$, then by Lemma 2.3(a) we have $g\left(-\frac{a}{2}+\frac{1}{b}\right)=0$; by the symmetry of $g$ this would imply that $g\left(\frac{a}{2}-\frac{1}{b}\right)=0$, which contradicts Lemma 2.1(b) with $x=-\frac{a}{2}$. Thus, we only have to deal with the case (2.7). If $G_{21}\left(x_{0}\right)=$ $G_{23}\left(x_{0}\right)=0$, then by Lemma $2.3(\mathrm{a})$, (b) we have

$$
g\left(x_{0}+\frac{1}{b}\right)=g\left(x_{0}+\frac{1}{b}+a\right)=g\left(x_{0}-\frac{1}{b}-a\right)=g\left(x_{0}-\frac{1}{b}\right)=0 .
$$

Inserting this information into the entries of the matrix $G\left(x_{0}\right)$ and applying Lemma 2.1 yield that

$$
\operatorname{det} G\left(x_{0}\right)=g\left(x_{0}+\frac{1}{b}-a\right) g\left(x_{0}\right) g\left(x_{0}-\frac{1}{b}+a\right)>0
$$

as desired. This completes the proof that $G(x)>0$ for $x \in\left[-\frac{a}{2}, 0\right]$. Since $g$ is symmetric around the origin, we have

$$
\begin{aligned}
\operatorname{det} G(-x) & =\operatorname{det}\left(g\left(-x-\frac{\ell}{b}+m a\right)\right)_{-1 \leq \ell, m \leq 1}=\operatorname{det}\left(g\left(x+\frac{\ell}{b}-m a\right)\right)_{-1 \leq \ell, m \leq 1} \\
& =-\operatorname{det}\left(g\left(x-\frac{\ell}{b}-m a\right)\right)_{-1 \leq \ell, m \leq 1}=\operatorname{det}\left(g\left(x-\frac{\ell}{b}+m a\right)\right)_{-1 \leq \ell, m \leq 1} \\
& =\operatorname{det} G(x) .
\end{aligned}
$$

Thus, $G(x)$ is also invertible for $\left.x \in] 0, \frac{a}{2}\right]$.

We are now ready to prove Theorem 1.2.

Proof of Theorem 1.2 By Corollary 2.4 and the continuity of $g$, $\inf _{x \in\left[-\frac{a}{2}, \frac{a}{2}\right]}|\operatorname{det} G(x)|>0$. We define $h$ on $\left[-\frac{3 a}{2}, \frac{3 a}{2}\right]$ by

$$
\left(\begin{array}{c}
h(x-a) \\
h(x) \\
h(x+a)
\end{array}\right)=G^{-1}(x)\left(\begin{array}{l}
0 \\
b \\
0
\end{array}\right), \quad x \in\left[-\frac{a}{2}, \frac{a}{2}\right],
$$

which is a bounded function. On $\mathbb{R} \backslash\left[-\frac{3 a}{2}, \frac{3 a}{2}\right]$, put $h(x)=0$. It follows immediately by definition of $h$ that then $g$ and $h$ are dual windows.

Remark 2.5 We note that this approach is tailored to the region of parameters $(a, b)$ in Theorem 1.2. For example, it does not apply to the region considered in Proposition 1.1(ii). 
In fact, if $0<b \leq \frac{2}{N+a}$, then the first row of $G(x)$ for $x \in\left[\frac{N}{2}-\frac{1}{b}+a, \frac{a}{2}\right]$ is the zero vector, and the third row of $G(x)$ for $x \in\left[-\frac{a}{2}, \frac{1}{b}-a-\frac{N}{2}\right]$ is the zero vector. Hence, we have $\inf _{x \in\left[-\frac{a}{2}, \frac{a}{2}\right]} \operatorname{det} G(x)=0$.

The conditions for $g \in V_{N, a}$ are technical. We will now give an example, showing that the conclusion about the size of the support of the dual window in Theorem 1.2 may break down if $g \notin V_{N, a}$.

Example 2.6 Let us consider the parameters $N=5, a=1$. Consider a symmetric and continuous function $g$ on $\mathbb{R}$ with supp $g=\left[-\frac{5}{2}, \frac{5}{2}\right]$; assume further that $g$ is increasing on $\left[-\frac{N}{2}, 0\right]=\left[-\frac{5}{2}, 0\right]$ and that

$$
g(0)=12, \quad g(-1)=10, \quad g\left(-\frac{4}{3}\right)=5, \quad g\left(-\frac{7}{3}\right)=3 .
$$

Then $\Delta_{a}^{2} g\left(-\frac{4}{3}\right)=g\left(-\frac{4}{3}\right)-2 g\left(-\frac{7}{3}\right)+g\left(-\frac{10}{3}\right)=-1<0$. Hence, $g$ does not satisfy (A3) at $x=-\frac{4}{3}$, that is, $g \notin V_{N, a}$.

We will show that, for $b=\frac{3}{N+2 a}=\frac{3}{7}$, there does not exist a bounded real-valued function $h \in L^{2}(\mathbb{R})$ with supp $h \subseteq\left[-\frac{3 a}{2}, \frac{3 a}{2}\right]$ such that the duality conditions (2.1) hold. In order to obtain a contradiction, let us assume that such a dual window indeed exists. Let $x_{0}=0$. Then

$$
\begin{aligned}
& x_{0}-a=-x_{0}-a=-1, \quad x_{0}-\frac{1}{b}+a=-x_{0}-\frac{1}{b}+a=-\frac{4}{3}, \\
& x_{0}-\frac{1}{b}=-x_{0}-\frac{1}{b}=-\frac{7}{3}, \quad x_{0}-\frac{1}{b}-a=-x_{0}-\frac{1}{b}-a=-\frac{10}{3},
\end{aligned}
$$

and, consequently,

$$
G\left(x_{0}\right)=\left(\begin{array}{ccc}
g\left(x_{0}+\frac{1}{b}-a\right) & g\left(x_{0}+\frac{1}{b}\right) & g\left(x_{0}+\frac{1}{b}+a\right) \\
g\left(x_{0}-a\right) & g\left(x_{0}\right) & g\left(x_{0}+a\right) \\
g\left(x_{0}-\frac{1}{b}-a\right) & g\left(x_{0}-\frac{1}{b}\right) & g\left(x_{0}-\frac{1}{b}+a\right)
\end{array}\right)=\left(\begin{array}{ccc}
5 & 3 & 0 \\
10 & 12 & 10 \\
0 & 3 & 5
\end{array}\right) .
$$

By the continuity of $g$ there exist continuous functions $\epsilon_{i j}(x), 1 \leq i, j \leq 3$, such that

$$
G(x)=\left(\begin{array}{ccc}
5+\epsilon_{11}(x) & 3+\epsilon_{12}(x) & \epsilon_{13}(x) \\
10+\epsilon_{21}(x) & 12+\epsilon_{22}(x) & 10+\epsilon_{23}(x) \\
\epsilon_{31}(x) & 3+\epsilon_{32}(x) & 5+\epsilon_{33}(x)
\end{array}\right)
$$

and

$$
\epsilon_{i j}(x) \rightarrow 0 \quad \text { as } x \rightarrow x_{0} .
$$

Then (2.2) implies that

$$
\left(\begin{array}{ccc}
5+\epsilon_{11}(x) & 3+\epsilon_{12}(x) & \epsilon_{13}(x) \\
10+\epsilon_{21}(x) & 12+\epsilon_{22}(x) & 10+\epsilon_{23}(x) \\
\epsilon_{31}(x) & 3+\epsilon_{32}(x) & 5+\epsilon_{33}(x)
\end{array}\right)\left(\begin{array}{c}
h(x-a) \\
h(x) \\
h(x+a)
\end{array}\right)=\left(\begin{array}{l}
0 \\
b \\
0
\end{array}\right)
$$


for a.e. $x \in\left[-\frac{a}{2}, \frac{a}{2}\right]$. By elementary row operations this leads to

$$
\left(\begin{array}{ccc}
5+\epsilon_{11}(x) & 3+\epsilon_{12}(x) & \epsilon_{13}(x) \\
\eta_{1}(x) & \eta_{2}(x) & \eta_{3}(x) \\
\epsilon_{31}(x) & 3+\epsilon_{32}(x) & 5+\epsilon_{33}(x)
\end{array}\right)\left(\begin{array}{c}
h(x-a) \\
h(x) \\
h(x+a)
\end{array}\right)=\left(\begin{array}{l}
0 \\
b \\
0
\end{array}\right), \quad \text { a.e. } x \in\left[-\frac{a}{2}, \frac{a}{2}\right]
$$

where $\eta_{i}(x):=\epsilon_{2 i}(x)-2 \epsilon_{1 i}(x)-2 \epsilon_{3 i}(x)$ for $1 \leq i \leq 3$. Since $h$ is a bounded function, ignoring a possible set of measure zero, this implies that

$$
b=\eta_{1}(x) h(x-a)+\eta_{2}(x) h(x)+\eta_{3}(x) h(x+a) \rightarrow 0
$$

as $x \rightarrow x_{0}$. This is a contradiction.

On the other hand, the condition $g \in V_{N, a}$ is not necessary for $\left\{E_{m b} T_{n a} g\right\}_{m, n \in \mathbb{Z}}$ to be a frame in the considered region. For example, let $N=1, a=\frac{1}{4}, b=2$ and take $g_{1}(x):=$ $\left(e^{-x^{2}}-e^{-\frac{1}{4}}\right) \chi_{\left[-\frac{1}{2}, \frac{1}{2}\right]}(x)$. Then elementary calculations show that (A3) does not hold for $x \in\left[-\frac{1}{10},-\frac{1}{16}\right] \subset\left[-\frac{N}{2},-\frac{N}{4}+\frac{3 a}{4}\right]$. But since $\operatorname{det} G(x)>0$ for $x \in\left[-\frac{a}{2}, \frac{a}{2}\right]$, we can prove that $\left\{E_{m b} T_{n a} g_{1}\right\}_{m, n \in \mathbb{Z}}$ is a frame by following the steps in the proof of Theorem 1.2.

\section{The set $V_{N, a}$}

In this section, we give easily verifiable sufficient conditions for a function $g$ to belong to $V_{N, a}$. Recall that a continuous function $f: \mathbb{R} \rightarrow \mathbb{C}$ with $\operatorname{supp} f=\left[-\frac{N}{2}, \frac{N}{2}\right]$ is piecewise continuously differentiable if there exist finitely many $x_{0}=-\frac{N}{2}<x_{1}<\cdots<x_{n}=\frac{N}{2}$ such that

(1) $f$ is continuously differentiable on $]-x_{i-1}, x_{i}[$ for every $i \in\{1, \ldots, n\}$;

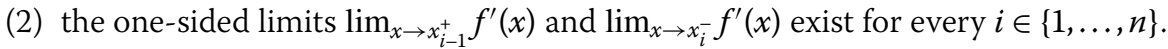

Note that if $g$ is a continuous and piecewise continuously differentiable function, then the fundamental theorem of calculus yields that

$$
\Delta_{a} g(x)=\int_{x-a}^{x} g^{\prime}(t) d t \quad \text { for all } x \in \mathbb{R}, a>0 .
$$

In order to avoid a tedious presentation, we will forego to mention the points where a piecewise continuously differentiable function is not differentiable, for example, in conditions (c) and (d) in the following Proposition 3.1. The result is inspired by explicit calculations for B-splines in Trebels and Steidl [26], Lemma 1.

Proposition 3.1 Let $N>0$ and assume that a continuous and piecewise continuously differentiable function $g: \mathbb{R} \rightarrow \mathbb{R}$ with $\operatorname{supp} g=\left[-\frac{N}{2}, \frac{N}{2}\right]$ satisfies the following conditions:

(a) $g$ is symmetric around the origin;

(b) $g$ is strictly increasing on $\left[-\frac{N}{2}, 0\right]$;

(c) $g^{\prime}$ is increasing on $\left.]-\frac{N}{2},-\frac{N}{4}\right]$;

(d) $g^{\prime}\left(-x-\frac{N}{2}\right) \leq g^{\prime}(x)$ for $x \in\left[-\frac{N}{4}, 0[\right.$.

Then $g \in \cap_{0<a<N} V_{N, a}$.

Proof Note that conditions (a) and (b) are exactly the same as (A1) and (A2). Thus, we will prove (A3). In the entire argument, we will assume that $g$ is differentiable; an elementary 
consideration then extends the result to the case of piecewise differentiable functions. Let us first consider $x \leq-\frac{N}{4}$. Then, by the mean value theorem,

$$
\Delta_{a}^{2} g(x)=a\left(\frac{g(x)-g(x-a)}{a}-\frac{g(x-a)-g(x-2 a)}{a}\right)=a\left(g^{\prime}(\xi)-g^{\prime}(\eta)\right)
$$

for some $\eta \in[x-2 a, x-a], \xi \in[x-a, x]$. Since $g^{\prime}$ is increasing up to $x=-\frac{N}{4}$, this proves that $\Delta_{a}^{2} g(x) \geq 0$ whenever $x \leq-\frac{N}{4}$.

We now consider $x \in\left[-\frac{N}{4}, \min \left(-\frac{N}{4}+\frac{3 a}{4}, 0\right)\right]$. Then

$$
x-a \leq \min \left(-\frac{N}{4}-\frac{a}{4},-a\right) \leq-\frac{N}{4}
$$

and

$$
\begin{aligned}
\Delta_{a}^{2} g(x)= & \int_{x-a}^{-\frac{N}{4}}\left(g^{\prime}(t)-g^{\prime}(t-a)\right) d t+\int_{-\frac{N}{4}}^{x}\left(g^{\prime}(t)-g^{\prime}(t-a)\right) d t \\
\geq & \int_{x-a}^{-\frac{N}{4}}\left(g^{\prime}(t)-g^{\prime}(t-a)\right) d t+\int_{-\frac{N}{4}}^{x}\left(g^{\prime}\left(-t-\frac{N}{2}\right)-g^{\prime}(t-a)\right) d t \\
= & g\left(-\frac{N}{4}\right)-g\left(-\frac{N}{4}-a\right)-g(x-a)+g(x-2 a) \\
& -g\left(-x-\frac{N}{2}\right)-g(x-a)+g\left(\frac{N}{4}-\frac{N}{2}\right)+g\left(-\frac{N}{4}-a\right) \\
= & g\left(-\frac{N}{4}\right)-g(x-a)-\left[g\left(-a-\frac{N}{4}\right)-g(x-2 a)\right] \\
& +g\left(-\frac{N}{4}\right)-g(x-a)-\left[g\left(-x-\frac{N}{2}\right)-g\left(-a-\frac{N}{4}\right)\right] .
\end{aligned}
$$

We now consider the terms (3.2) and (3.3) separately. For (3.2), by the mean value theorem,

$$
\begin{aligned}
& g\left(-\frac{N}{4}\right)-g(x-a)-\left[g\left(-a-\frac{N}{4}\right)-g(x-2 a)\right] \\
& \quad=\left(-\frac{N}{4}-x+a\right)\left(\frac{g\left(-\frac{N}{4}\right)-g(x-a)}{-\frac{N}{4}-x+a}-\frac{g\left(-a-\frac{N}{4}\right)-g(x-2 a)}{-\frac{N}{4}-x+a}\right) \\
& \quad=\left(-\frac{N}{4}-x+a\right)\left(g^{\prime}(\xi)-g^{\prime}(\eta)\right)
\end{aligned}
$$

for some $\eta \in\left[x-2 a,-a-\frac{N}{4}\right], \xi \in\left[x-a,-\frac{N}{4}\right]$. Since $-a-\frac{N}{4} \leq x-a$, we have $\eta \leq \xi \leq-\frac{N}{4}$; thus, by assumption (c), $g^{\prime}(\eta) \leq g^{\prime}(\xi)$. Recalling that $x \leq-\frac{N}{4}+\frac{3 a}{4}<-\frac{N}{4}+a$, we have $-\frac{N}{4}-$ $x+a>0$; thus, we conclude that the term in (3.2) indeed is nonnegative.

For (3.3), we consider two cases. If $x-a \geq-x-\frac{N}{2}$, then exactly the same argument as for (3.2) works. If $x-a<-x-\frac{N}{2}$, then we perform the same argument after a rearrangement of the terms. Indeed,

$$
\begin{aligned}
& g\left(-\frac{N}{4}\right)-g(x-a)-\left[g\left(-x-\frac{N}{2}\right)-g\left(-a-\frac{N}{4}\right)\right] \\
& \quad=g\left(-\frac{N}{4}\right)-g\left(-x-\frac{N}{2}\right)-\left[g(x-a)-g\left(-a-\frac{N}{4}\right)\right]
\end{aligned}
$$




$$
\begin{aligned}
& =\left(x+\frac{N}{4}\right)\left(\frac{g\left(-\frac{N}{4}\right)-g\left(-x-\frac{N}{2}\right)}{x+\frac{N}{4}}-\frac{g(x-a)-g\left(-a-\frac{N}{4}\right)}{x+\frac{N}{4}}\right) \\
& =\left(x+\frac{N}{4}\right)\left(g^{\prime}(\xi)-g^{\prime}(\eta)\right)
\end{aligned}
$$

for some $\eta \in\left[-a-\frac{N}{4}, x-a\right], \xi \in\left[-x-\frac{N}{2},-\frac{N}{4}\right]$. As before, this implies that (3.3) is nonnegative.

We now consider $x=-\frac{N}{4}+\frac{3 a}{4}$; according to (1.2), we must prove that

$$
g\left(\frac{N}{4}-\frac{3 a}{4}\right)-2 g\left(-\frac{N}{4}-\frac{a}{4}\right) \geq 0
$$

First, if $a>\frac{2 N}{3}$ (i.e., $\left.-\frac{N}{4}+\frac{3 a}{4}>\frac{N}{4}\right)$, then we have

$$
\begin{aligned}
& g\left(\frac{N}{4}-\frac{3 a}{4}\right)-2 g\left(-\frac{N}{4}-\frac{a}{4}\right) \\
& \quad=\frac{N-a}{2}\left(\frac{g\left(\frac{N}{4}-\frac{3 a}{4}\right)-g\left(-\frac{N}{4}-\frac{a}{4}\right)}{\frac{N-a}{2}}-\frac{g\left(-\frac{N}{4}-\frac{a}{4}\right)-g\left(\frac{-3 N}{4}+\frac{a}{4}\right)}{\frac{N-a}{2}}\right) \\
& \quad=\frac{N-a}{2}\left(g^{\prime}(\xi)-g^{\prime}(\eta)\right)
\end{aligned}
$$

for some $\xi \in\left[-\frac{N}{4}-\frac{a}{4}, \frac{N}{4}-\frac{3 a}{4}\right], \eta \in\left[-\frac{3 N}{4}+\frac{a}{4},-\frac{N}{4}-\frac{a}{4}\right]$; thus, (3.4) holds by assumption (c).

Now assume that $\frac{N}{2}<a \leq \frac{2 N}{3}$; then $-\frac{N}{4}+\frac{3 a}{4} \leq \frac{N}{4}$, and

$$
-\frac{N}{4} \leq \frac{N}{4}-\frac{3 a}{4} \leq 0, \quad-\frac{N}{4}-\frac{a}{4}<-\frac{N}{2}+\frac{a}{4}<0
$$

Thus,

$$
\begin{aligned}
& g\left(\frac{N}{4}-\frac{3 a}{4}\right)-2 g\left(-\frac{N}{4}-\frac{a}{4}\right) \\
& \quad \geq g\left(-\frac{N}{4}\right)-g\left(-\frac{N}{4}-\frac{a}{4}\right)-\left(g\left(-\frac{N}{4}-\frac{a}{4}\right)-g\left(-\frac{N}{4}-\frac{2 a}{4}\right)\right) ;
\end{aligned}
$$

this can again be expressed in terms of the difference $g^{\prime}(\xi)-g^{\prime}(\eta)$ with $\xi \in\left[-\frac{N}{4}-\frac{a}{4},-\frac{N}{4}\right]$, $\eta \in\left[-\frac{N}{4}-\frac{2 a}{4},-\frac{N}{4}-\frac{a}{4}\right]$ and is hence positive.

Finally, we assume that $\frac{N}{3} \leq a \leq \frac{N}{2}$. Then $-\frac{N}{4}+\frac{3 a}{4} \leq \frac{N}{4}$; since $-\frac{N}{4}-\frac{a}{4}<-\frac{a}{2}$, assumption (b) implies that

$$
g\left(\frac{N}{4}-\frac{3 a}{4}\right)-g\left(-\frac{N}{4}-\frac{a}{4}\right) \geq g\left(\frac{N}{4}-\frac{3 a}{4}\right)-g\left(-\frac{a}{2}\right) \geq \int_{-\frac{a}{2}}^{\frac{N}{4}-\frac{3 a}{4}} g^{\prime}(t) d t=:(*) .
$$

Since $-\frac{N}{4} \leq-\frac{a}{2}<\frac{N}{4}-\frac{3 a}{4} \leq 0$, (d) implies that

$$
(*) \geq \int_{-\frac{a}{2}}^{\frac{N}{4}-\frac{3 a}{4}} g^{\prime}\left(-t-\frac{N}{2}\right) d t=\int_{-\frac{3 N}{4}+\frac{3 a}{4}}^{-\frac{N}{2}+\frac{a}{2}} g^{\prime}(t) d t=:(* *) .
$$


Since $-\frac{N}{2}+\frac{a}{2} \leq-\frac{N}{4}$ and $g^{\prime}$ is increasing on $\left.]-\infty,-\frac{N}{4}\right]$, shifting the integration interval by $-\frac{N}{4}+\frac{3 a}{4} \geq 0$ to the left yields that

$$
(* *) \geq \int_{-\frac{N}{2}}^{-\frac{N}{4}-\frac{a}{4}} g^{\prime}(t) d t=g\left(-\frac{N}{4}-\frac{a}{4}\right)
$$

thus, (3.4) holds, as desired.

Proposition 3.1 immediately leads to the following simple criterion for a function to belong to $\bigcap_{0<a<N} V_{N, a}$.

Corollary 3.2 Let $N>0$ and assume that a continuous and piecewise continuously differentiable function $g: \mathbb{R} \rightarrow \mathbb{R}$ with $\operatorname{supp} g=\left[-\frac{N}{2}, \frac{N}{2}\right]$ satisfies the following conditions:

(a) $g$ is symmetric around the origin;

(b) $g^{\prime}$ is positive and increasing on $]-\frac{N}{2}, 0[$.

Then $g \in \bigcap_{0<a<N} V_{N, a}$.

We will now describe several functions belonging to $V_{N, a}$, either for all $a$ from $] 0, N[$ or from a subinterval hereof.

Example 3.3 Consider the B-splines $B_{N}, N \in \mathbb{N}$, defined recursively by

$$
B_{1}=\chi_{[-1 / 2,1 / 2]}, \quad B_{N+1}=B_{N} * B_{1} .
$$

In [26], Lemma 1 , it is proved that, for $N \in \mathbb{N} \backslash\{1\}$,

(i) $B_{N}^{\prime}$ is increasing on $\left.]-\frac{N}{2},-\frac{N}{4}+\frac{1}{4}\right]$;

(ii) $B_{N}^{\prime}\left(-x-\frac{N}{2}\right) \leq B_{N}^{\prime}(x)$ for $x \in\left[-\frac{N}{4}, 0[\right.$.

Thus, Proposition 3.1 implies that $B_{N} \in \bigcap_{0<a<N} V_{N, a}$ for $N \in \mathbb{N} \backslash\{1\}$.

Example 3.4 Let $N \in \mathbb{N} \backslash\{1\}$ and define

$$
f_{N}(x):=\cos ^{2 N-2}\left(\frac{\pi x}{N}\right) \chi_{\left[-\frac{N}{2}, \frac{N}{2}\right]}(x)
$$

Direct calculations show that, for $\left.x \in]-\frac{N}{2},-\frac{N}{4}\right]$,

$$
f_{N}^{\prime \prime}(x)=\frac{(2 N-2) \pi^{2}}{N^{2}} \cos ^{2 N-4}\left(\frac{\pi x}{N}\right)\left((2 N-3) \sin ^{2}\left(\frac{\pi x}{N}\right)-\cos ^{2}\left(\frac{\pi x}{N}\right)\right) \geq 0
$$

and, for $x \in\left[-\frac{N}{4}, 0[\right.$,

$$
f_{N}^{\prime}(x)-f_{N}^{\prime}\left(-x-\frac{N}{2}\right)=\frac{(2 N-2) \pi}{2 N} \sin \left(\frac{2 \pi x}{N}\right)\left(\sin ^{2 N-4}\left(\frac{\pi x}{N}\right)-\cos ^{2 N-4}\left(\frac{\pi x}{N}\right)\right) \geq 0 .
$$

By Proposition 3.1, $f_{N} \in \bigcap_{0<a<N} V_{N, a}$.

In the following examples we consider continuous and compactly supported 'variants' of the two-sided exponential function, the Gaussian, and other classical functions. 
Example 3.5 Let $N>0$ and define

$$
h_{N}(x):=\left(e^{-|x|}-e^{-\frac{N}{2}}\right) \chi_{\left[-\frac{N}{2}, \frac{N}{2}\right]}(x) .
$$

Then $h_{N} \in \bigcap_{0<a<N} V_{N, a}$ by Corollary 3.2.

Example 3.6 Let $N>0$ and define

$$
k_{N}(x):=\left(\frac{1}{1+|x|}-\frac{1}{1+\frac{N}{2}}\right) \chi_{\left[-\frac{N}{2}, \frac{N}{2}\right]}(x) .
$$

Then $k_{N} \in \bigcap_{0<a<N} V_{N, a}$ by Corollary 3.2.

In the following examples simple sufficient conditions in Proposition 3.1 and Corollary 3.2 are not satisfied. We will use the definition directly to show that the considered functions belong to $V_{N, a}$ for certain ranges of the parameter $a$.

Example 3.7 Let $N>0$ and consider

$$
p_{N}(x):=\left(\frac{1}{1+x^{2}}-\frac{1}{1+\left(\frac{N}{2}\right)^{2}}\right) \chi_{\left[-\frac{N}{2}, \frac{N}{2}\right]}(x) .
$$

We will show that

(a) $p_{N} \in \bigcap_{\frac{3 N}{7} \leq a<N} V_{N, a}$;

(b) $p_{N} \in \bigcap_{\frac{N}{3} \leq a<\frac{3 N}{7}} V_{N, a}$ if $N \geq \sqrt{\frac{12}{5}} \approx 1.5451 \cdots$.

It is clear that (A1) and (A2) hold, so for the considered values of $a$, we now check (A3). In fact, we will prove more, namely that

$$
\Delta_{a}^{2} p_{N}(x) \geq 0, \quad x \in\left[-\frac{N}{2},-\frac{N}{4}+\frac{3 a}{4}\right] .
$$

Considering any $a \in\left[\frac{N}{3}, N[\right.$, we have

$$
-\frac{N}{2}<-\frac{N}{4}+\frac{3 a}{4}<\frac{N}{2}, \quad a-\frac{N}{2}<-\frac{N}{4}+\frac{3 a}{4}<a+\frac{N}{2}, \quad-\frac{N}{4}+\frac{3 a}{4}<2 a-\frac{N}{2} ;
$$

so the fact that $p_{N}>0$ on $]-\frac{N}{2}, \frac{N}{2}\left[, p_{N}(\cdot-a)>0\right.$ on $] a-\frac{N}{2}, a+\frac{N}{2}\left[\right.$, and $p_{N}(\cdot-2 a)>0$ on ]2a- $\frac{N}{2}, 2 a+\frac{N}{2}[$ immediately shows that

(1) $\left.\left.p_{N}(x) \neq 0, x \in\right]-\frac{N}{2},-\frac{N}{4}+\frac{3 a}{4}\right]$;

(2) $\left.\left.p_{N}(x-a)=0, x \in\left[-\frac{N}{2}, a-\frac{N}{2}\right] ; p_{N}(x-a) \neq 0, x \in\right] a-\frac{N}{2},-\frac{N}{4}+\frac{3 a}{4}\right]$;

(3) $p_{N}(x-2 a)=0, x \in\left[-\frac{N}{2},-\frac{N}{4}+\frac{3 a}{4}\right]$.

Thus,

$$
\Delta_{a}^{2} p_{N}(x)= \begin{cases}p_{N}(x), & x \in\left[-\frac{N}{2},-\frac{N}{2}+a\right], \\ p_{N}(x)-2 p_{N}(x-a), & \left.x \in]-\frac{N}{2}+a,-\frac{N}{4}+\frac{3 a}{4}\right] .\end{cases}
$$

Since $p_{N}(x) \geq 0, x \in\left[-\frac{N}{2},-\frac{N}{2}+a\right]$, it now suffices to prove that

$$
\left.\left.p_{N}(x)-2 p_{N}(x-a) \geq 0, \quad x \in\right]-\frac{N}{2}+a,-\frac{N}{4}+\frac{3 a}{4}\right] \text {. }
$$


Let $\left.x \in]-\frac{N}{2}+a,-\frac{N}{4}+\frac{3 a}{4}\right]$. We see that

$$
\begin{aligned}
p_{N}(x)-2 p_{N}(x-a) & =p_{N}(x)-p_{N}(x-a)-p_{N}(x-a) \\
& =\frac{1}{1+x^{2}}-\frac{1}{1+(x-a)^{2}}-\left(\frac{1}{1+(x-a)^{2}}-\frac{1}{1+\left(\frac{N}{2}\right)^{2}}\right) \\
& =\frac{(x-a)^{2}-x^{2}}{\left(1+x^{2}\right)\left(1+(x-a)^{2}\right)}-\frac{\left(\frac{N}{2}\right)^{2}-(x-a)^{2}}{\left(1+(x-a)^{2}\right)\left(1+\left(\frac{N}{2}\right)^{2}\right)} .
\end{aligned}
$$

In order to prove (a), we now assume that $\frac{3 N}{7} \leq a<N$. Since $x^{2} \leq\left(\frac{N}{2}\right)^{2}$ and $(x-a)^{2}-x^{2} \geq$ 0 , we have

$$
\begin{aligned}
p_{N}(x)-2 p_{N}(x-a) & \geq \frac{(x-a)^{2}-x^{2}}{\left(1+\left(\frac{N}{2}\right)^{2}\right)\left(1+(x-a)^{2}\right)}-\frac{\left(\frac{N}{2}\right)^{2}-(x-a)^{2}}{\left(1+(x-a)^{2}\right)\left(1+\left(\frac{N}{2}\right)^{2}\right)} \\
& =\frac{h(x)}{\left(1+\left(\frac{N}{2}\right)^{2}\right)\left(1+(x-a)^{2}\right)},
\end{aligned}
$$

where

$$
h(x):=2(x-a)^{2}-x^{2}-\frac{N^{2}}{4}=(x-2 a)^{2}-2 a^{2}-\frac{N^{2}}{4} .
$$

Note that the quadratic function $h$ is symmetric around $x=2 a$. Since $-\frac{N}{4}+\frac{3 a}{4}<2 a$ and $\frac{3 N}{7} \leq a<N$, we have

$$
h(x) \geq h\left(-\frac{N}{4}+\frac{3 a}{4}\right)=\frac{1}{16}(N-a)(7 a-3 N) \geq 0 .
$$

Thus,

$$
p_{N}(x)-2 p_{N}(x-a) \geq 0 \text {. }
$$

Therefore, (3.5) holds, that is, the proof of (a) is completed.

In order to prove (b), assume now that $N \geq \sqrt{\frac{12}{5}}$ and $\frac{N}{3} \leq a<\frac{3 N}{7}$. Since $(x-a)^{2}-x^{2}=$ $2 a\left(\frac{a}{2}-x\right)$ and $\left(\frac{N}{2}\right)^{2}-(x-a)^{2}=\left(\frac{N}{2}-x+a\right)\left(\frac{N}{2}+x-a\right)$, (3.7) implies that

$$
p_{N}(x)-2 p_{N}(x-a)=\frac{1}{1+(x-a)^{2}}\left(\frac{2 a\left(\frac{a}{2}-x\right)}{1+x^{2}}-\frac{\left(\frac{N}{2}-x+a\right)\left(\frac{N}{2}+x-a\right)}{1+\left(\frac{N}{2}\right)^{2}}\right) .
$$

Using $-\frac{N}{2}+a<x \leq-\frac{N}{4}+\frac{3 a}{4}$, it follows that $\frac{N}{2}-x+a<N$ and $\frac{N}{2}+x-a \leq \frac{N}{4}-\frac{a}{4} \leq-x+\frac{a}{2}$; thus,

$$
\begin{aligned}
p_{N}(x)-2 p_{N}(x-a) & \geq \frac{1}{1+(x-a)^{2}}\left(\frac{2 a\left(\frac{a}{2}-x\right)}{1+x^{2}}-\frac{N\left(\frac{a}{2}-x\right)}{1+\left(\frac{N}{2}\right)^{2}}\right) \\
& =\frac{\frac{a}{2}-x}{1+(x-a)^{2}}\left(\frac{q(x)}{\left(1+x^{2}\right)\left(1+\left(\frac{N}{2}\right)^{2}\right)}\right)
\end{aligned}
$$


where $q(x):=2 a\left(1+\left(\frac{N}{2}\right)^{2}\right)-N\left(1+x^{2}\right)$. Since $\frac{N}{3} \leq a<\frac{3 N}{7}$ and $-\frac{N}{2}+a<x \leq-\frac{N}{4}+\frac{3 a}{4}$, we have $-\frac{N}{6}<x \leq \frac{N}{14}$; so $x^{2}<\frac{N^{2}}{36}$. Thus,

$$
q(x) \geq 2 \frac{N}{3}\left(1+\left(\frac{N}{2}\right)^{2}\right)-N\left(1+\frac{N^{2}}{36}\right)=N\left(-\frac{1}{3}+\frac{5 N^{2}}{36}\right) \geq 0 .
$$

Thus, $p_{N}(x)-2 p_{N}(x-a) \geq 0$. Therefore, (3.5) holds, as desired.

Example 3.8 Let $N>0$ and consider

$$
g_{N}(x):=\left(e^{-x^{2}}-e^{-\frac{N^{2}}{4}}\right) \chi_{\left[-\frac{N}{2}, \frac{N}{2}\right]}(x)
$$

We will show that $g_{N} \in \bigcap_{\frac{3 N}{7} \leq a<N} V_{N, a}$. As in Example 3.7 (see (3.6)), it suffices to check that

$$
\left.\left.g_{N}(x)-2 g_{N}(x-a) \geq 0, \quad x \in\right]-\frac{N}{2}+a,-\frac{N}{4}+\frac{3 a}{4}\right] .
$$

Let $\left.x \in]-\frac{N}{2}+a,-\frac{N}{4}+\frac{3 a}{4}\right]$, and $a \in\left[\frac{3 N}{7}, N[\right.$. We see that

$$
\begin{aligned}
g_{N}(x)-2 g_{N}(x-a) & =g_{N}(x)-g_{N}(x-a)-g_{N}(x-a) \\
& =e^{-x^{2}}-e^{-(x-a)^{2}}-\left(e^{-(x-a)^{2}}-e^{-\frac{N^{2}}{4}}\right) \\
& =e^{-(x-a)^{2}}\left(e^{(x-a)^{2}-x^{2}}-1\right)-e^{-\frac{N^{2}}{4}}\left(e^{\frac{N^{2}}{4}-(x-a)^{2}}-1\right) .
\end{aligned}
$$

Since $-(x-a)^{2} \geq-\frac{N^{2}}{4}$ and $(x-a)^{2}-x^{2} \geq 0$, we have

$$
\begin{aligned}
g_{N}(x)-2 g_{N}(x-a) & \geq e^{-\frac{N^{2}}{4}}\left(e^{(x-a)^{2}-x^{2}}-1\right)-e^{-\frac{N^{2}}{4}}\left(e^{\frac{N^{2}}{4}-(x-a)^{2}}-1\right) \\
& =e^{-\frac{N^{2}}{4}}\left(e^{(x-a)^{2}-x^{2}}-e^{\frac{N^{2}}{4}-(x-a)^{2}}\right) \\
& =e^{-(x-a)^{2}}\left(e^{h(x)}-1\right),
\end{aligned}
$$

where $h(x):=2(x-a)^{2}-x^{2}-\left(\frac{N}{2}\right)^{2}$. From (3.8) we have $h(x) \geq 0$. Thus,

$$
g_{N}(x)-2 g_{N}(x-a) \geq 0,
$$

as desired.

\section{Competing interests}

The authors declare that they have no competing interests.

\section{Authors' contributions}

All authors contributed equally to this work. All authors read and approved the final manuscript.

\section{Author details}

'Department of Applied Mathematics and Computer Science, Technical University of Denmark, Building 303, Lyngby, 2800, Denmark. ${ }^{2}$ Division of General Studies, UNIST, 50 UNIST-gil, Ulsan, 44919, Republic of Korea. ${ }^{3}$ Department of Mathematics, Yeungnam University, 280 Daehak-Ro, Gyeongsan, Gyeongbuk 38541, Republic of Korea. 


\section{Acknowledgements}

The authors would like to thank the reviewers for many useful suggestions, which clearly improved the presentation. In particular, one reviewer suggested to formulate condition (A3) for membership of $V_{N, a}$ in terms of second-order differences, which is much more transparent than our original condition. He also suggested the approach in the current proof of Proposition 3.1, which is shorter than our original one. This research was supported by Basic Science Research Program through the National Research Foundation of Korea (NRF) funded by the Ministry of Education (2013R1A1A2A10011922).

\section{Received: 25 November 2015 Accepted: 16 February 2016 Published online: 18 March 2016}

\section{References}

1. Feichtinger, HG, Kaiblinger, N: Varying the time-frequency lattice of Gabor frames. Trans. Am. Math. Soc. 356, 2001-2023 (2004)

2. Ascensi, G, Feichtinger, HG, Kaiblinger, N: Dilation of the Weyl symbol and the Balian-Low theorem. Trans. Am. Math Soc. $366,3865-3880(2014)$

3. Lyubarskii, Y: Frames in the Bargmann space of entire functions. Adv. Sov. Math. 11, 167-180 (1992)

4. Seip, K: Density theorems for sampling and interpolation in the Bargmann-Fock space I. J. Reine Angew. Math. 429, 91-106 (1992)

5. Seip, K, Wallsten, R: Density theorems for sampling and interpolation in the Bargmann-Fock space II. J. Reine Angew. Math. 429, 107-113 (1992)

6. Janssen, AJEM, Strohmer, T: Hyperbolic secants yield Gabor frames. Appl. Comput. Harmon. Anal. 12, 259-267 (2002)

7. Janssen, AJEM: Some Weyl-Heisenberg frame bound calculations. Indag. Math. 7, 165-183 (1996)

8. Janssen, AJEM: On generating tight Gabor frames at critical density. J. Fourier Anal. Appl. 9, 175-214 (2003)

9. Gröchenig, K, Stöckler, J: Gabor frames and totally positive functions. Duke Math. J. 162, 1003-1031 (2013)

10. Janssen, AJEM: Zak transforms with few zeros and the tie. In: Feichtinger, HG, Strohmer, T (eds.) Advances in Gabor Analysis. Appl. Numer. Harmon. Anal., pp. 31-70. Birkhäuser, Boston (2003)

11. Dai, XR, Sun, Q: The abc-problem for Gabor systems. Mem. Am. Math. Soc. (to appear)

12. Del Prete, V: Estimates, decay properties, and computation of the dual function for Gabor frames. J. Fourier Anal. Appl. 5, 545-562 (1999)

13. Laugesen, RS: Gabor dual spline windows. Appl. Comput. Harmon. Anal. 27, 180-194 (2009)

14. Kloos, T, Stöckler, J: Zak transforms and Gabor frames of totally positive functions and exponential B-splines. J. Approx. Theory 184, 209-237 (2014)

15. Kim, I: Gabor frames with trigonometric spline dual windows. Asian-Eur. J. Math. 8(4), 1550072 (2015)

16. Gröchenig, K, Janssen, AJEM, Kaiblinger, N, Pfander, G: Note on B-splines, wavelet scaling functions, and Gabor frames. IEEE Trans. Inf. Theory 49, 3318-3320 (2003)

17. Christensen, $\mathrm{O}, \mathrm{Kim}, \mathrm{HO}, \mathrm{Kim}, \mathrm{RY}$ : On entire functions restricted to intervals, partition of unities, and dual Gabor frames. Appl. Comput. Harmon. Anal. 38, 72-86 (2015)

18. Christensen, $\mathrm{O}, \mathrm{Kim}, \mathrm{HO}, \mathrm{Kim}, \mathrm{RY}$ : Gabor windows supported on $[-1,1]$ and compactly supported dual windows. Appl. Comput. Harmon. Anal. 28, 89-103 (2010)

19. Christensen, O, Kim, HO, Kim, RY: Gabor windows supported on $[-1,1]$ and dual windows with small support. Adv. Comput. Math. 36, 525-545 (2012)

20. Christensen, $\mathrm{O}, \mathrm{Kim}, \mathrm{HO}$, Kim, RY: On Gabor frames generated by sign-changing windows and B-splines. Appl. Comput. Harmon. Anal. 39, 534-544 (2015)

21. Lemvig, J, Nielsen, H: Counterexamples to the B-spline conjecture for Gabor frames. J. Fourier Anal. Appl. (to appear)

22. Prete, VD: Estimates, decay properties, and computation of the dual function for Gabor frames. J. Fourier Anal. Appl. 5, 545-561 (1999)

23. Gröchenig, K: Partitions of unity and new obstructions for Gabor frames. Preprint

24. Lyubarskii, Y, Nes, PG: Gabor frames with rational density. Appl. Comput. Harmon. Anal. 34, 488-494 (2013)

25. Christensen, O: An Introduction to Frames and Riesz Bases, 2nd expanded edn. Birkhäuser, Boston (2016)

26. Trebels, B, Steidl, G: Riesz bounds of Wilson bases generated by B-splines. J. Fourier Anal. Appl. 6, 171-184 (2000)

27. Ron, A, Shen, Z: Weyl-Heisenberg systems and Riesz bases in $L^{2}\left(\mathbb{R}^{d}\right)$. Duke Math. J. 89, 237-282 (1997)

28. Janssen, AJEM: The duality condition for Weyl-Heisenberg frames. In: Feichtinger, HG, Strohmer, T (eds.) Gabor Analysis and Algorithms: Theory and Applications, pp. 33-84. Birkhäuser, Boston (1998) 